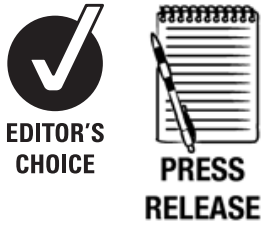

1 Institute for Cardiovascular Pharmacology and Epidemiology, Mahlow, Germany

2DBM GmbH, Mühlhausen,

Germany

${ }^{3}$ Klinikum Lippe $\mathrm{GmbH}$, Fachbereich Herz-Kreislauf, Detmold, Germany

${ }^{4}$ Kreiskrankenhaus Leer, Leer, Germany

${ }^{5}$ Klinikum Hildesheim $\mathrm{GmbH}$, Hildesheim, Germany ${ }^{6}$ Herz-Zentrum Bad Krozingen, Bad Krozingen, Germany ${ }^{7}$ Stiftung Institut für Herzinfarktforschung Ludwigshafen an der Universität Heidelberg, Ludwigshafen, Germany

\section{Correspondence to}

Professor Dr med Ulrich Tebbe, Klinikum Lippe $\mathrm{GmbH}$, Fachbereich Herz-Kreislauf, Röntgenstrasse 18, 32756 Detmold, Germany: ulrich.tebbe@klinikum-lippe.de

Accepted 15 December 2009

\title{
The effect of optimal medical therapy on 1-year mortality after acute myocardial infarction
}

\author{
P Bramlage, ${ }^{1}$ C Messer, ${ }^{2}$ N Bitterlich, ${ }^{2}$ C Pohlmann, ${ }^{1}$ A Cuneo, ${ }^{3}$ E Stammwitz, \\ J Tebbenjohanns, ${ }^{5}$ H Gohlke, $^{6}$ J Senges, ${ }^{7}$ U Tebbe ${ }^{3}$
}

\begin{abstract}
Objectives Five drug classes have been shown to improve the prognosis of acute myocardial infarction in clinical trials: aspirin, $\beta$-blockers, statins, renin angiotensin system (RAS) blockers and thienopyridines. We aimed to assess whether the benefits of combining these drugs (termed optimal medical therapy, OMT), will result in a reduction of mortality in clinical practice.

Design Nationwide registry

Setting Hospitals with a cardiology unit or internal medicine department.
\end{abstract}

Patients 5353 patients with acute myocardial infarction. At hospital discharge 89\% received aspirin, 90\% $\beta$-blockers, $84 \%$ statins, 81\% RAS blockers, 70\% a thienopyridine and $46.2 \%$ OMT

Interventions Pharmacotherapy

Main outcome measures OR with $95 \% \mathrm{Cl}$ for mortality from myocardial infarction were calculated and adjusted for patient risk at baseline.

Results Total mortality was reduced by $74 \%$ in patients receiving OMT (adj OR 0.26; $95 \% \mathrm{Cl} 0.18$ to 0.38 ) versus patients receiving one or no drug. This was consistent in subgroups defined by STEMI/NSTEMI, diabetes and gender. Mortality was also reduced in patients receiving 2-4 drugs (adj OR 0.49; 95\% $\mathrm{Cl} 0.35$ to 0.68 ), diabetic patients being the only subgroup with no significant effect. Analyses on the relative importance of either component revealed that withdrawal of $\beta$-blockers ladj OR $0.63 ; 95 \% \mathrm{Cl} 0.34$ to 1.16 ) and/or a combination of aspirin/clopidogrel (adj OR 0.59; 95\% Cl 0.20 to 1.17) abolished the risk reduction conferred by OMT.

Conclusions OMT over 1 year was associated with a significantly lower mortality of patients with acute myocardial infarction in clinical practice. However OMT is provided to less than half of eligible patients leaving room for substantial improvement.

\section{INTRODUCTION}

Acute myocardial infarction (AMI) is a major cause of morbidity and mortality. Community studies conducted before the advent of percutaneous coronary intervention, thrombolysis and refined pharmacotherapy have consistently shown overall case fatality rates of about $50 \%$ in the first month, half of these occurring within the first 2 hours after initial presentation. ${ }^{1}$

While out-of-hospital mortality has changed little over the past few years, in-hospital and longterm mortality of patients surviving until hospital admission is on the decline. ${ }^{2}$ Recent studies have shown an overall 1 -month mortality of $4-6 \%{ }^{34}$ In the TRITON TIMI 38 trial the overall death rate in patients with acute coronary syndromes after 15 months was as low as $3.1 \%{ }^{5}$
For the secondary prevention of vascular events clinical trials have shown that five drug classes substantially improve the prognosis of patients with acute myocardial infarction: aspirin, $\beta$ blockers, statins, renin angiotensin system (RAS) blockers and thienopyridines. Optimal medical therapy (OMT) consisting of these five drug classes has the potential to substantially lower long-term mortality. The use of these drugs in clinical practice is however less than optimal with $89 \%$ receiving aspirin, 90\% $\beta$-blockers, 84\% statins, 81\% RAS blockers and $70 \%$ a thienopyridine at hospital discharge in the recent SAMI (Secondary prevention after Acute Myocardial Infarction) registry. ${ }^{6}$ After 1 year the prescription rate was substantially lower. On the other hand using OMT is of particular importance for clinical practice where $11 \%$ have recurrent myocardial infarction within 1 year and mortality remains much higher than in the general population with a RR of 2.1 (95\% CI 1.9 to 2.5) for the first 30 days and 5.6 (95\% CI 5.1 to 6.2 ) thereafter. ${ }^{7}$ Further, it is of importance since patients in clinical practice are at an increased overall cardiovascular risk than those in clinical trials. 8

We therefore analysed the proportion of patients being treated with OMT in clinical practice and its impact on mortality overall and in subgroups defined by the presence of ST deviations (STEMI vs NSTEMI), diabetes and gender.

\section{METHODS}

SAMI was a nationwide registry in 79 hospitals with a cardiology unit or an internal medicine department in Germany. Methods have been described in detail previously. ${ }^{6}$ In short, SAMI was initiated and performed as a prospective multicentre study conducted by the ALKK-study group (Arbeitsgemeinschaft Leitender Kardiologischer Krankenhausärzte). The registry study design was approved and authorised by the ethics commission of the Ärztekammer Westfalen-Lippe and the Westfälische Wilhelms Universität Münster. Patients were consecutively documented at admission, hospital discharge and after a follow-up of 1 year. Oral but not written informed consent, because of the non-interventional design of the registry, was obtained before inclusion in the study.

\section{Study population}

Between February 2003 and October 2004 a total of 5353 consecutive patients hospitalised for an episode of acute ST-elevation myocardial infarction (STEMI) or acute non-ST-elevation MI 
(NSTEMI, symptoms lasting $<24$ hours) according to ESC (European Society of Cardiology) definition were enrolled. Further definitions: (1) cardiogenic shock was defined as any of the following: hypotension with systolic blood pressure $<90 \mathrm{~mm} \mathrm{Hg}$ as a result of reduced left ventricular ejection fraction with a need for catecholamines. Signs of left ventricular failure with dyspnoea and signs of congestion. (2) Heart failure was defined as any of the following: dyspnoea with signs of congestion. Peripheral oedema was regarded as a sign of right heart failure.

The procedures of documented diagnostic measures were defined as well (ECG at rest, ejection fraction (EF), diagnostic tests in ischaemia, coronary angiography).

\section{Pharmacotherapy}

Optimal medical therapy (OMT) in this paper is defined as receiving $\mathrm{ACE}$ inhibitors/ angiotensin-receptor blockers (ARBs), $\beta$-blockers, statins, aspirin, clopidogrel unless contraindicated. In case one component was contraindicated, patients receiving all indicated therapies were optimally treated from a clinical perspective but were not included in the current analysis. Practice guidelines at the time of SAMI recommended the use of aspirin and $\beta$-blockers in all patients with acute coronary syndrome (ACS) without contraindications. ${ }^{9-11}$ All patients were considered to be eligible for antiplatelet/anticoagulant therapy unless they had a history of life-threatening bleeding, coagulopathy or thrombocytopenia. All patients were considered to be eligible for $\beta$-blockers unless they had significant bradycardia (heart rate $<50$ beats/min) or hypotension (systolic blood pressure $<90 \mathrm{~mm} \mathrm{Hg}$ ). ACE inhibitors or ARBs were considered to be indicated in all patients with STEMI and in patients with non-ST-elevation ACS who had hypertension, diabetes or heart failure. Contraindications to ACE inhibitors were hypotension and severe renal dysfunction (serum creatinine $>221 \mu \mathrm{mol} / 1$ in men or $>177 \mu \mathrm{mol} / 1$ in women). Because accumulating evidence suggests no threshold below which lipidmodifying medication is ineffective and contraindications are rare, all patients with ACS were considered to be eligible for statin therapy. ${ }^{12} 13$

\section{Data collection}

Baseline demographic data, previous medication, patients' status, early diagnostic procedures, risk factors/concomitant diseases and therapeutic procedural data were obtained at hospital admission. The intra-hospital clinical course and diagnostic procedures, final diagnosis, secondary preventive steps, complications and patient status were recorded as hospital discharge. After 1 year patient status was explored including cardiovascular events and current pharmacotherapy by patient questionnaires.

\section{Statistical analysis}

The collected data were verified with respect to formal and contextual accuracy depending on performed measurement variance and logical dependence. In the case of absent or inconclusive information hospitals were contacted for further data. The completeness of collected variables was between $95 \%$ and 99\%, except for 'manifestation of infarction' (86.4\%) and some laboratory data during the acute phase $(77.3 \%-96.0 \%)$. The biometric evaluation of the data was performed in a descriptive and exploratory way. For continuous variables the number of patients, average value, and $\mathrm{SD}$, median, minimum and maximum were determined. For categorical variables percentage frequencies were determined. OR were calculated with $95 \%$ CI and adjusted for age, cardiac arrest on presentation, heart rate, systolic blood pressure, Killip class, ST-segment deviation, abnormal cardiac biomarkers, serum creatinine, previous myocardial infarction (MI), heart failure and inhospital revascularisation.

\section{RESULTS}

A total of 5353 patients with acute myocardial infarction were included into the SAMI registry. Eighty-four per cent received statins, $89 \%$ aspirin, 70\% clopidogrel, 90\% $\beta$-blockers and $81 \%$ RAS-blocking agents at hospital discharge. Overall, $46.2 \%$ of patients received OMT (figure 1). Patients receiving OMT were younger, more frequently male, had more risk factors like nicotine abuse, hypertension and dyslipidaemia and had more often received $\mathrm{CABG}$ in the past (table 1 ). On the other hand a history
Figure 1 Prescription of drugs having been shown to be effective in the secondary prevention of vascular events after myocardial infarction (\% of all patients eligible for drug prescription). CSE, cholesterol synthesis enzyme inhibitors; RAS, renin angiotensin system.

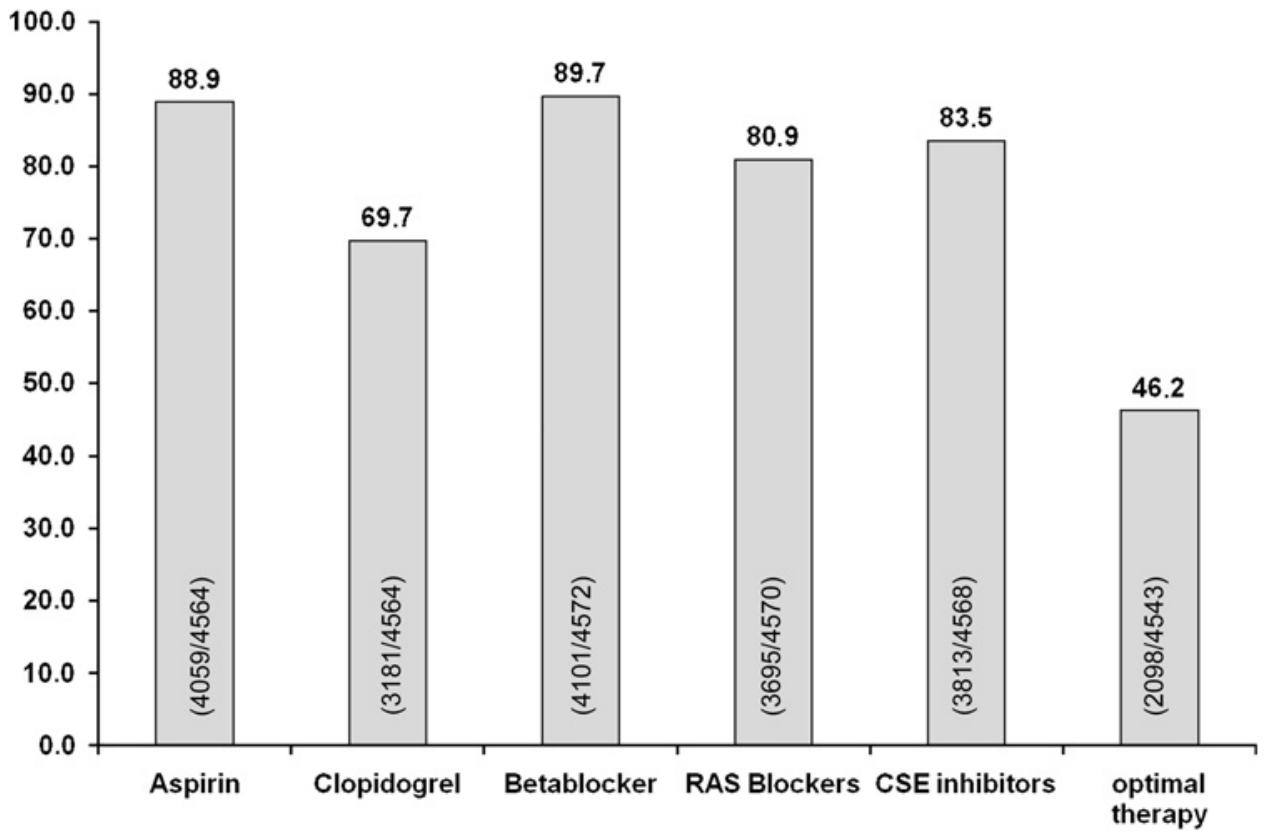


Table 1 Baseline demographic and clinical characteristics

\begin{tabular}{|c|c|c|c|c|}
\hline & Optimal therapy at discharge $(\mathrm{n} / \mathrm{N})$ & Suboptimal therapy at discharge $(\mathrm{n} / \mathrm{N})$ & p Value & No or one drug at discharge $(\mathrm{n} / \mathrm{N})$ \\
\hline Age (median, 25,75 th percentile) & $(66.3 ; 56.9 ; 75.1)(1977)$ & $(70.5 ; 60.9 ; 79.1)(3166)$ & $<0.001$ & $(73.2 ; 63.8 ; 81.1)(314)$ \\
\hline Male (\%) & $69.7 \%(1378 / 1977)$ & $63.7 \%(2019 / 3169)$ & $<0.001$ & $63.4 \%(199 / 314)$ \\
\hline \multicolumn{5}{|l|}{ Type of ACS } \\
\hline NSTEMI (\%) & $45.4 \%(849 / 1871)$ & $46.2 \%(1414 / 3058)$ & 0.555 & $41.7 \%(126 / 302)$ \\
\hline \multicolumn{5}{|l|}{ Concomitant risk factors } \\
\hline Nicotine (\%) & $38.9 \%(766 / 1968)$ & $29.9 \%(944 / 3157)$ & $<0.001$ & $24.9 \%(78 / 313)$ \\
\hline Diabetes (\%) & $28.6 \%(563 / 1968)$ & $29.6 \%(934 / 3157)$ & 0.454 & $30.7 \%(96 / 313)$ \\
\hline \multicolumn{5}{|l|}{ Concomitant disease } \\
\hline Previous MI (\%) & $21.0 \%(416 / 1977)$ & $19.8 \%(629 / 3172)$ & 0.293 & $20.4 \%(64 / 314)$ \\
\hline Previous stroke (\%) & $5.7 \%(112 / 1977)$ & $8.7 \%(276 / 3172)$ & $<0.001$ & $11.5 \%(36 / 314)$ \\
\hline Previous PAD (\%) & $6.9 \%(136 / 1977)$ & $9.0 \%(286 / 3172)$ & 0.007 & $12.1 \%(38 / 314)$ \\
\hline$\beta$-blockers (\%) & $36.7 \%(726 / 1977)$ & $29.6 \%(938 / 3172)$ & $<0.001$ & $25.2 \%(79 / 314)$ \\
\hline RAS-blocking agents (\%) & $34.1 \%(674 / 1977)$ & $28.2 \%(893 / 3172)$ & $<0.001$ & $24.5 \%(77 / 314)$ \\
\hline Statins (\%) & $22.5 \%(444 / 1977)$ & $13.2 \%(420 / 3172)$ & $<0.001$ & $10.2 \%(32 / 314)$ \\
\hline
\end{tabular}

of stroke or known PAD was more frequent in patients not receiving OMT (suboptimal therapy at discharge). There was a higher frequency of previous stroke $(11.5 \%$ vs $5.7 \%$ in patients with OMT) and/or PAD (12.1\% vs $6.9 \%)$ in this patient subgroup.

\section{In-hospital management}

Patients not receiving optimal medical therapy at discharge more often showed complications such as heart failure and resuscitation at admission $(28.0 \%$ and $7.6 \%$, respectively) or during the course of the hospitalisation $(25.7 \%$ and $4.2 \%$, respectively). More patients having the LAD stented received OMT (37.7\% vs $33.1 \%, \mathrm{p}=0.003)$. For details see table 2 .

\section{OMT AND 1-YEAR MORTALITY}

Figure 2 shows that OMT was strongly associated with a better survival 1 year after acute myocardial infarction. The OR for mortality was lower in patients receiving OMT (adj OR 0.260; $95 \%$ CI 0.179 to 0.379$)$ compared to no or one drug $(\mathrm{p}<0.001)$.
The effect of OMT was consistent in subgroups of patients defined by the type of MI (STEMI vs NSTEMI), the presence or absence of diabetes and in both genders.

Mortality was also reduced in patients receiving 2-4 drugs (adj OR 0.486 ; $95 \%$ CI 0.346 to 0.684 ) compared to no or one drug $(\mathrm{p}<0.001)$, the effect was nominal but the CI overlapped indicating non-significance. This trend was observed in every subgroup of patients defined. In patients with diabetes the effect of 2-4 drugs was not significantly reduced against $0-1$ drugs $(\mathrm{p}=0.111)$.

In figure 3 the relative importance of OMT components on mortality after AMI is displayed. The effect of OMT was still significantly preserved after withdrawal of either RAS-blocking agents, clopidogrel and a statin with CI overlapping with the one of OMT. The withdrawal of $\beta$-blockers (adj OR 0.627; $95 \%$ CI 0.339 to 1.156 ) and of the aspirin/clopidogrel combination (adj OR 0.428; 95\% CI 0.199 to 1.170) however resulted in a non-significant lowering of mortality compared to using only no or one drug.

Table 2 In-hospital management

\begin{tabular}{|c|c|c|c|}
\hline & Optimal therapy at discharge & Suboptimal therapy at discharge & p Value \\
\hline Pre-hospital time $\leq 3$ hours & $42.0 \%(724 / 1725)$ & $44.4 \%(1159 / 2610)$ & 0.113 \\
\hline \multicolumn{4}{|l|}{ Complications at admission } \\
\hline Heart failure (\%) & $17.4 \%(340 / 1953)$ & $28.0 \%(875 / 3122)$ & $<0.001$ \\
\hline Cardiogenic shock (\%) & $3.1 \%(61 / 1959)$ & $11.3 \%(355 / 3136)$ & $<0.001$ \\
\hline Resuscitation (\%) & $2.6 \%(50 / 1951)$ & $7.6 \%(239 / 3128)$ & $<0.001$ \\
\hline Lysis $\leq 1$ hour $(\%)$ & $60.1 \%(125 / 208)$ & $79.0 \%(361 / 457)$ & $<0.001$ \\
\hline PTCA $\leq 3$ hour $(\%)$ & $62.6 \%(531 / 848)$ & $63.2 \%(532 / 842)$ & 0.809 \\
\hline LAD (\%) & $37.7 \%(628 / 1664)$ & $33.1 \%(657 / 1985)$ & 0.003 \\
\hline $\mathrm{RCX}(\%)$ & $18.0 \%(300 / 1664)$ & $18.0 \%(357 / 1985)$ & 0.973 \\
\hline RCA (\%) & $30.0 \%(500 / 1664)$ & $29.5 \%(585 / 1985)$ & 0.704 \\
\hline Mean ejection fraction (\%) & $56.6(907)$ & $56.0(1053)$ & 0.565 \\
\hline \multicolumn{4}{|l|}{ In-hospital complications } \\
\hline Resuscitation (\%) & $1.7 \%(31 / 1853)$ & $4.2 \%(121 / 2885)$ & $<0.001$ \\
\hline
\end{tabular}


Figure 2 Univariate and multivariate analysis -independent predictors of 1-year mortality overall, STEMI/NSTEMI, diabetes/non-diabetes, male/female. Adj OR, adjusted OR (adjustment for age, cardiac arrest on presentation, heart rate, systolic blood pressure, Killip class, ST-segment deviation, abnormal cardiac biomarker, serum creatinine, previous $\mathrm{MI}$ and heart failure and in-hospital revascularisation); cruOR, myocardial infarction; OMT, optimal medical therapy; STEMI, ST-elevation myocardial infarction. crude OR; NSTEMI, non-ST-elevation

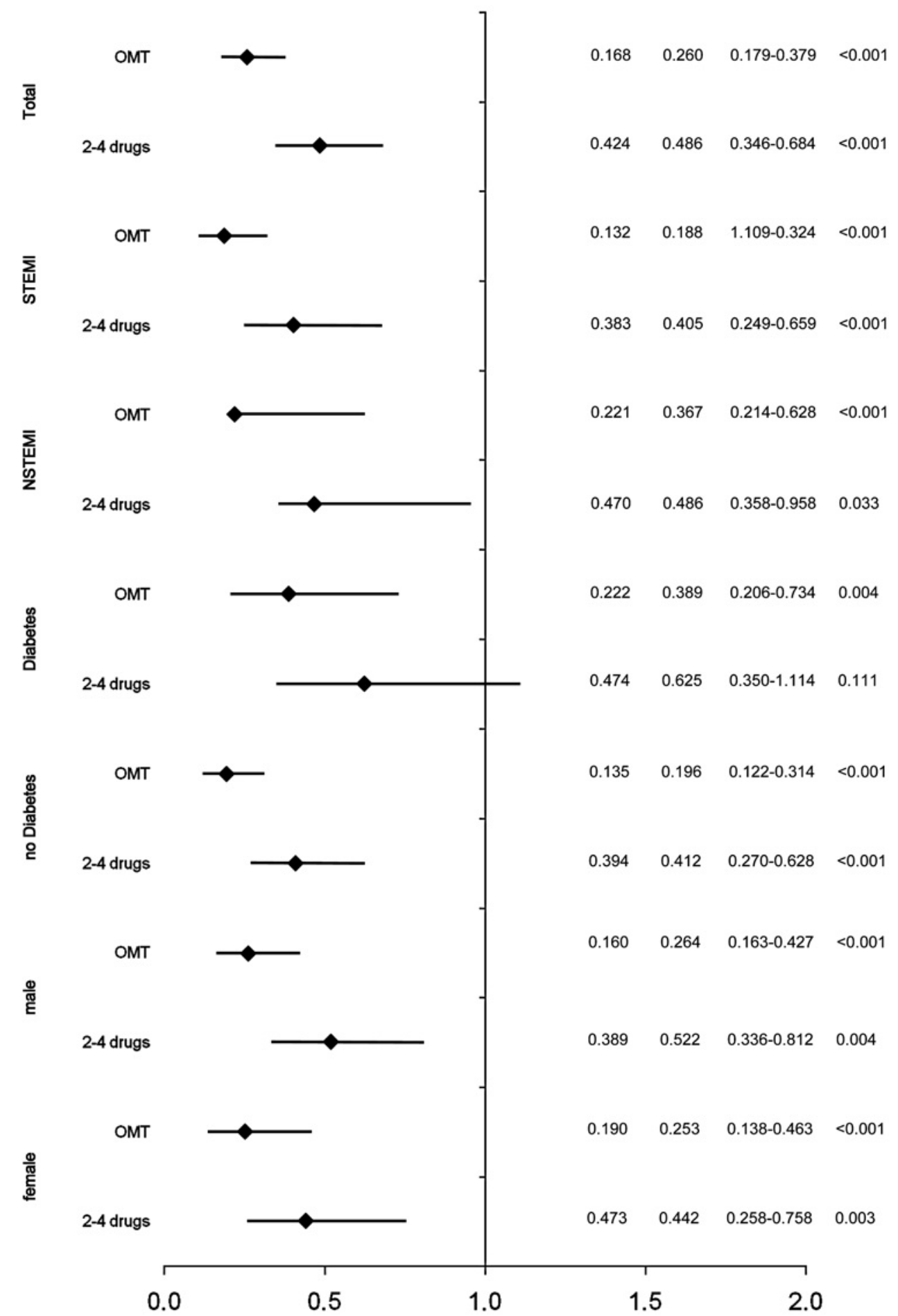

\section{DISCUSSION}

The secondary prevention of cardiovascular and cerebrovascular events as well as mortality is a key effort in the medical management of acute myocardial infarction. Long-term survival has been substantially improved over the past few years, but mortality rates in clinical practice are still higher than in randomised controlled trials. SAMI was able to document that use of OMT was associated with an increased 1-year survival. Total mortality was reduced by $74 \%$ in patients receiving OMT, which was consistent in subgroups of patients defined by STEMI/ NSTEMI, diabetes and gender. However less than $50 \%$ of patients received such an optimal combination of all five drug classes, leaving substantial room for improvement.

\section{Optimal medical therapy use}

The majority of patients received aspirin, $\beta$-blockers, statins, RAS-blocking agents or clopidogrel at hospital discharge. Although the initial prescription rates of single components of OMT were encouraging, a subset of these patients discontinued treatment during the course of follow-up: At 1 year $84.0 \%$ of patients received $\beta$-blockers ( $90 \%$ at hospital discharge), $67.5 \%$ ACE inhibitors and $11.7 \%$ ARBs $(81 \%$ at least one of them at 
Figure 3 Optimal medical therapy (OMT) and the effect of withdrawing specific components. adjOR, adjusted OR (adjustment for age, cardiac arrest on presentation, heart rate, systolic blood pressure, Killip class, ST-segment deviation, abnormal cardiac biomarker, serum creatinine, previous $\mathrm{Ml}$ and heart failure and in-hospital revascularisation); ARBs, angiotensin receptor blockers; ASA, aspirin; cruOR, crude OR; OMT, optimal medical therapy.

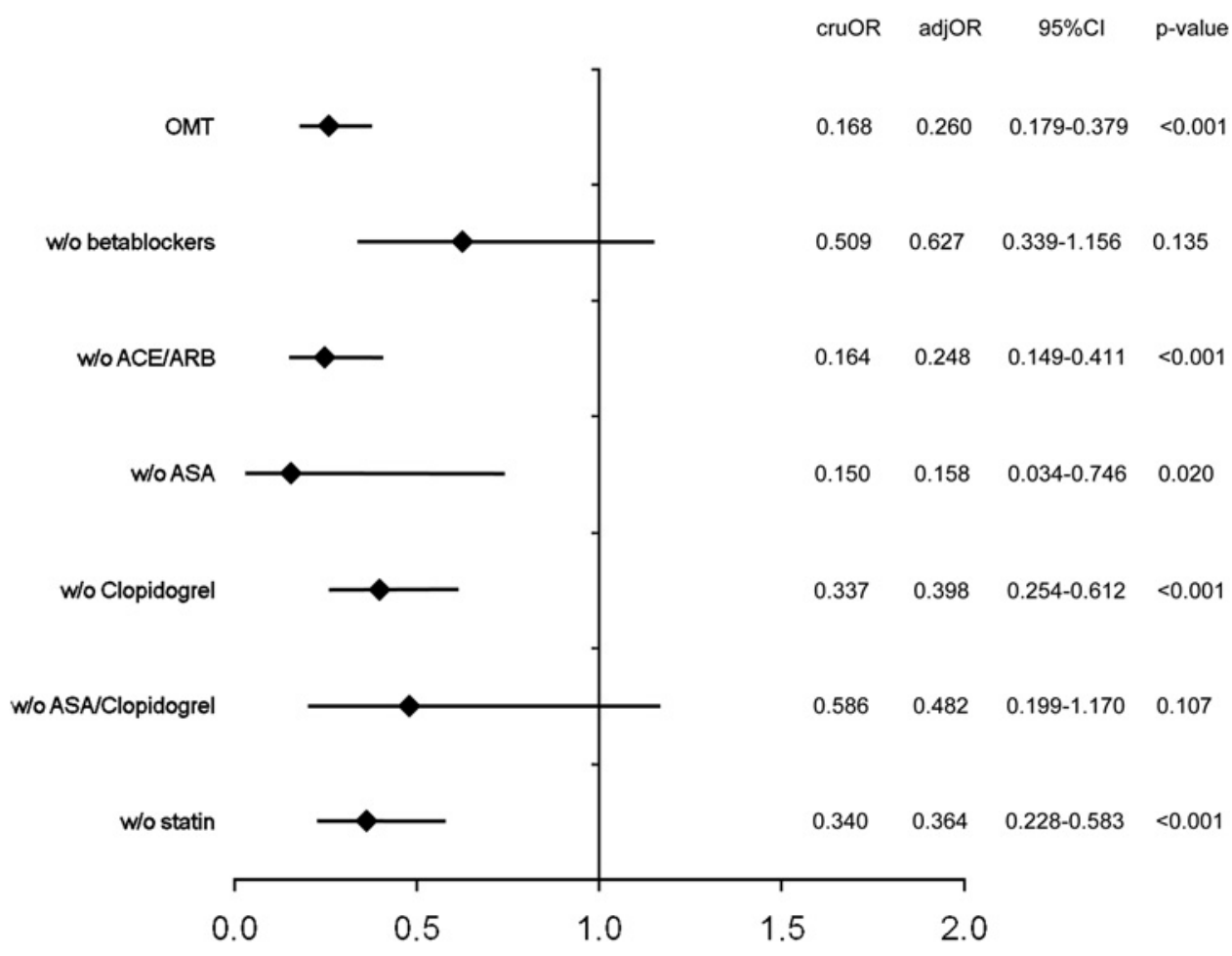

discharge), $87.7 \%$ either clopidogrel or aspirin (89\% aspirin, 70\% clopidogrel) and $76.8 \%$ lipid-lowering agents (84\%). Unfortunately we were, for example, not able to follow the decline in clopidogrel and provide a mean duration of prescription, but the transition of patients from the initial hospitalisation back into primary care may represent a critical transition point. At this point adequate long-term medication has to be ensured. ${ }^{11}{ }^{14-16}$

A further aspect to consider in evaluating the benefits of optimal medical therapy is within-class differences in the riskbenefit ratio of drugs and that the use of high doses might provide additional benefit in organ protection. Examples of this are available for thienopyridines, for which different ratios of benefits and risk have been reported per drug and drug dose, ${ }^{5}$ for the choice of ACE inhibitors/ARBs with a differential effect on morbidity and mortality ${ }^{17}$ and for statins for which a different potential to lower cholesterol values has been reported.

\section{Optimal medical therapy}

There are an abundant number of studies documenting the underuse of evidence-based pharmacotherapy in the secondary prevention of vascular events after AMI. ${ }^{14}{ }^{18-22}$ However there are far fewer studies documenting the role of optimal medical therapy on long-term survival in clinical practice. ${ }^{11} 23-25$

In the most recent report, Yan et al documented OMT use in $51.8 \%$ of patients in the Canadian ACS II registry (2002-3), which is compatible with our data. ${ }^{11}$ They further documented an absolute increase of $23 \%$ over the previous Canadian ACS I registry (1999-2001) at which time $28.9 \%$ of patients received OMT $(p<0.001)$.

In their registry OMT use (all five drug classes) was associated with an $46 \%$ risk reduction (adj OR of 0.54 ) in comparison with using no or one drug. The use of two or three drugs was again associated with a less pronounced but still significant risk reduction of $35 \%$ vs no or one drug (OR 0.65). Both comparisons were highly significant. The effect size in the Canadian registry pointed into the same direction with on average greater reduction in mortality in SAMI. Furthermore the proportion of high-risk patients in SAMI receiving OMT was higher than that in the two Canadian registries.

\section{Limitations}

The present dataset describes the course and outcome of a large sample of patients with either STEMI or NSTEMI in 79 hospitals throughout Germany and thus gives a pretty complete picture of clinical practice in Germany. Inherent in the observational study design, however, are a number of restrictions. Since this is not a randomised trial subjects who are more likely to get OMT may differ in variables (health awareness, exercise, social status) not obtained with the clinical case report form and bias arising from these differences can not be accounted for. Further to this, groups under consideration have been formed on the basis of variables that are themselves prone to bias. Therefore there is no possibility of differentiating whether differences in therapy led to a different outcome or patient characteristics leading to a particular choice of therapy might have influenced this outcome. Finally the analysis presented in this paper may be compromised by below optimal completeness of data (85\%). Data for critical endpoints were however available in $95 \%$ of cases, making a systematic distortion of results unlikely.

\section{Conclusions}

Secondary prevention of cardiovascular events is a cornerstone of the long-term prognosis of acute myocardial infarction. OMT with statins, aspirin, clopidogrel, $\beta$-blockers, ACE inhibitors/ ARBs over 1 year was crude and, after adjusting for confounding baseline variables, associated with a significantly lower mortality. It is however prescribed in less than $50 \%$ of cases and every effort should be made to increase its use.

Acknowledgements We acknowledge the active participation of 79 hospitals and their staff throughout Germany. A full list of participating hospitals has been published. ${ }^{6}$

Funding The study has been funded by Solvay Pharma.

Competing interests SAMI was funded by an unrestricted educational grant from Solvay Arzneimittel, Hannover, Germany. Solvay had no active role in the preparation 
of neither this analysis nor the decision to publish the manuscript. UT, JS and PB have been actively involved in advisory boards and have received research grants of different pharmaceutical companies producing cardiovascular drugs for patients with acute myocardial infarction. The statistical analyses were performed by NB who is a named author on this manuscript.

Ethics approval This study was conducted with the approval of the Ärztekammer Westfalen-Lippe and the Westfälische Wilhelms Universität Münster.

Provenance and peer review Not commissioned; not externally peer reviewed.

\section{REFERENCES}

1. Tunstall-Pedoe $\mathbf{H}$, Kuulasmaa $\mathrm{K}$, Mahonen $\mathrm{M}$, et al. Contribution of trends in survival and coronary-event rates to changes in coronary heart disease mortality: 10 -year results from 37 WHO MONICA project populations. Monitoring trends and determinants in cardiovascular disease. Lancet 1999;353:1547-57.

2. Goldberg RJ, Glatfelter K, Burbank-Schmidt $\mathrm{E}$, et al. Trends in community mortality due to coronary heart disease. Am Heart J 2006;151:501-7.

3. Assessment of the Safety and Efficacy of a New Treatment Strategy with Percutaneous Coronary Intervention (ASSENT-4 PCI) investigators. Primary versus tenecteplase-facilitated percutaneous coronary intervention in patients with ST-segment elevation acute myocardial infarction (ASSENT-4 PCI): randomised trial. Lancet 2006;367:569-78.

4. Armstrong PW, Granger CB, Adams PX, et al. Pexelizumab for acute ST-elevation myocardial infarction in patients undergoing primary percutaneous coronary intervention: a randomized controlled trial. JAMA 2007;297:43-51.

5. Wiviott SD, Braunwald E, McCabe $\mathrm{CH}$, et al. Prasugrel versus clopidogrel in patients with acute coronary syndromes. N Engl J Med 2007;357:2001-15.

6. Tebbe U, Messer C, Stammwitz E, et al. [Reduction of in-hospital mortality and improved secondary prevention after acute myocardial infarction. First results from the registry of secondary prevention after acute myocardial infarction (SAMI)]. Dtsch Med Wochenschr 2007:132:1559-66.

7. Buch P, Rasmussen S, Gislason GH, et al. Temporal decline in the prognostic impact of a recurrent acute myocardial infarction 1985 to 2002. Heart 2007;93:210-15.

8. Fox KA, Dabbous $\mathrm{OH}$, Goldberg RJ, et al. Prediction of risk of death and myocardial infarction in the six months after presentation with acute coronary syndrome: prospective multinational observational study (GRACE). BMJ 2006;333:1091.

9. Braunwald $\mathbf{E}$, Antman EM, Beasley JW, et al. ACC/AHA guideline update for the management of patients with unstable angina and non-ST-segment elevation myocardial infarction-2002: summary article: a report of the American college of cardiology/American heart association task force on practice guidelines (Committee on the Management of Patients With Unstable Angina). Circulation 2002:106:1893-900

10. Antman EM, Anbe DT, Armstrong PW, et al. ACC/AHA guidelines for the management of patients with ST-elevation myocardial infarction-executive summary: a report of the American college of cardiology/American heart association task force on practice guidelines (Writing Committee to Revise the 1999 Guidelines for the Management of Patients With Acute Myocardial Infarction). Can J Cardiol 2004;20:977-1025

11. Yan AT, Yan RT, Tan M, et al. Optimal medical therapy at discharge in patients with acute coronary syndromes: temporal changes, characteristics, and 1-year outcome. Am Heart J 2007;154:1108-15.

12. Wiviott SD, Cannon CP, Morrow DA, et al. Can low-density lipoprotein be too low? The safety and efficacy of achieving very low low-density lipoprotein with intensive statin therapy: a PROVE IT-TIMI 22 substudy. J Am Coll Cardiol 2005:46:1411-16.

13. Heart Protection Study Collaborative Group. MRC/BHF Heart Protection Study of cholesterol lowering with simvastatin in 20,536 high-risk individuals: a randomised placebo-controlled trial. Lancet 2002;360:7-22.

14. Ho PM, Spertus JA, Masoudi FA, et al. Impact of medication therapy discontinuation on mortality after myocardial infarction. Arch Intern Med 2006;166:1842-7.

15. Mehta RH, Montoye CK, Faul J, et al. Enhancing quality of care for acute myocardial infarction: shifting the focus of improvement from key indicators to process of care and tool use: the American college of cardiology acute myocardial infarction guidelines applied in practice project in Michigan: Flint and Saginaw Expansion. J Am Coll Cardiol 2004;43:2166-73.

16. Heidenreich PA. Patient adherence: the next frontier in quality improvement. $\mathrm{Am} \mathrm{J}$ Med 2004;117:130-2.

17. Pitt B, Segal R, Martinez FA, et al. Randomised trial of losartan versus captopril in patients over 65 with heart failure (Evaluation of Losartan in the Elderly Study, ELITE). Lancet 1997;349:747-52.

18. Roe MT, Peterson ED, Newby LK, et al. The influence of risk status on guideline adherence for patients with non-ST-segment elevation acute coronary syndromes. Am Heart J 2006;151:1205-13.

19. Granger CB, Steg PG, Peterson E, et al. Medication performance measures and mortality following acute coronary syndromes. Am J Med 2005;118:858-65.

20. Eagle KA, Kline-Rogers E, Goodman SG, et al. Adherence to evidence-based therapies after discharge for acute coronary syndromes: an ongoing prospective observational study. Am J Med 2004;117:73-81.

21. Blackburn DF, Dobson RT, Blackburn JL, et al. Adherence to statins, beta-blockers and angiotensin-converting enzyme inhibitors following a first cardiovascular event: a retrospective cohort study. Can J Cardiol 2005;21:485-8.

22. Gislason GH, Rasmussen JN, Abildstrom SZ, et al. Long-term compliance with beta-blockers, angiotensin-converting enzyme inhibitors, and statins after acute myocardial infarction. Eur Heart J 2006;27:1153-8.

23. Bonello L, De LA, Roy $P$, et al. Impact of optimal medical therapy and revascularization on outcome of patients with chronic kidney disease and on dialysis who presented with acute coronary syndrome. Am J Cardiol 2008:102:535-40.

24. Mukherjee D, Fang J, Chetcuti S, et al. Impact of combination evidence-based medical therapy on mortality in patients with acute coronary syndromes. Circulation 2004:109:745-9.

25. Allen LA, O'Donnell CJ, Giugliano RP, et al. Care concordant with guidelines predicts decreased long-term mortality in patients with unstable angina pectoris and non-ST-elevation myocardial infarction. Am J Cardiol 2004;93:1218-22. 